\title{
Increasing Breast Cancer Systemic Therapy Use Before Surgery in the United States: Scaling Down and the Promise of Selective Elimination of Surgery
}

\author{
Henry M. Kuerer, MD, PhD, FACS \\ PH and Fay Etta Robinson Distinguished Professor of Surgery and Cancer Research, Executive Director, Breast Programs, \\ MD Anderson Cancer Network, Division of Surgery, Department of Breast Surgical Oncology, The University of Texas \\ MD Anderson Cancer Center, Houston, TX
}

We are in a vastly different new era in our understanding of the biology of breast cancer and the efficacy of systemic therapies. Together with this information and the imperative of integration of multidisciplinary approaches for the management of breast cancer, over the past two decades the benefits and potential pitfalls on how to integrate and optimize neoadjuvant systemic therapy (NST) with surgery and radiotherapy has been elucidated. ${ }^{1-8}$ Simply stated, if the molecular subtype and clinical prognostic stage of a breast cancer at diagnosis predicts that the patient will have a survival benefit with systemic therapy, then the best approach might be to administer the therapy before surgery if doing so can allow for increased patient benefits. These benefits are striking and unequivocally proven to allow for less breast and axillary nodal surgery with corresponding minimization of morbidity, while maintaining the same overall and disease-free survival benefit of adjuvant systemic therapy. It should also be noted that within molecular subtype groupings, there is interest and continued study of de-escalation of systemic therapies based on risk of recurrence and prognostic stage.

A pathologic complete response (pCR) with NST is associated with a disease-free and overall survival benefit compared with not having a pCR. ${ }^{4-9-11}$ Patients with HER 2+/triple-negative breast cancer (TNBC) subtypes have the highest rate of $\mathrm{pCR}$ rates compared with luminal

(C) Society of Surgical Oncology 2018

First Received: 18 May 2018;

Published Online: 26 July 2018

H. M. Kuerer, MD, PhD, FACS

e-mail: hkuerer@mdanderson.org type A/B cancers. ${ }^{10,11}$ The introduction and testing of both paclitaxel and anti-HER2+ agents are now routinely incorporated for patients with TNBC and HER2+ tumors, respectively, ${ }^{12}$ and this has led to increasing rates of pCR. ${ }^{13}$ Another valuable benefit of delivering systemic therapy prior to surgery is that it gives the patient and oncologist detailed information about their particular tumor with respect to the efficacy of the particular regimen they are receiving. Although, progression of disease while receiving NST rarely happens, it allows the clinical team to consider other agents or trials to identify an effective regimen for the patient's particular tumor, and this theoretically may positively influence their outcome. Finally, there is now randomized trial evidence that receiving additional adjuvant therapy after identification of patients with residual breast cancer after NST can increase their survival from breast cancer compared with patients who did not receive additional therapy. In a recent landmark trial, capecitabine administered based on identifying residual disease after NST was associated with a significant absolute increase of $8.5 \%$ in the overall survival of patients with HER2-negative breast cancer compared with patients randomly assigned to no further therapy. ${ }^{14}$

Given this background information, it is not surprising that there is now new definitive information regarding increasing use of NST in these particular subtypes of breast cancer in the US. ${ }^{15}$ In this issue of Annals of Surgical Oncology, Murphy et al. utilized the US National Cancer Database (NCDB) to determine when chemotherapy was received, and found that 251,726 patients $(79.8 \%)$ received adjuvant chemotherapy and 63,538 patients (20.2\%) received NST. ${ }^{15}$ Although significant increases in NST use were seen in all biologic subtypes, the greatest proportions 
and increases in NST use were seen among TNBC $(19.5-33.7 \%)$ and HER $2+$ (HR-/HER2 + 21.5-39.8\%; HR+/HER2+ 17.0-33.7\%) tumors. Within these subtypes, Murphy et al. found use of NST significantly increased in all stages in TNBC, with the largest increase seen in stage II disease (stage I: $3.4-12.6 \%$; stage II: $24.0-44.8 \%$; and stage III: 53.6-60.3\%). Within the HER2+ groups, significant increases in the use of NST were mostly seen in stage I and II disease (HR+/HER2+: stage I: 3.7-13.3\%, stage II: $22.6-49.4 \%$, stage III: 46.2-54.5\%; HR-/ HER2+: stage I: $3.0-17.4 \%$, stage II: $25.2-52.4 \%$, stage III not significant: $54.3-54.9 \%$ ). Interestingly for HER2+ and TNBC, initially clinical node-positive patients were more likely than node-negative cases to receive NST, but this changed in the later time periods when NST use in nodenegative cases was similar or greater than in node-positive cases. The authors note that guideline recommendations from the National Comprehensive Cancer Network and the St. Gallen International Expert Consensus for use of NST for HER2+ and TNBC are consistent with their findings, however there remains opportunity for increasing the use of NST in the US. ${ }^{16}$

It is essential and critical to take all perspectives into view when proclaiming recommendations for patient care. In this regard, there has been a long-term concern that we do not place the patient at increased risk while receiving NST versus adjuvant systemic therapy. Approximately 20-30 years ago, as the practice of NST was being tested and increasingly utilized, there was concern that this approach might lead to increased local regional recurrence (LRR) among patients treated with breast-conserving therapy. ${ }^{1}$ Later, two important meta-analyses showed that LRR significantly increased in patients receiving NST compared with adjuvant systemic therapy when surgery was omitted and the patients received only radiotherapy without surgery. ${ }^{17,18}$ Unfortunately, this type of concern persists based on another recent meta-analysis that showed a 10-year absolute $3.2 \%$ increase in LRR among patients receiving NST versus adjuvant systemic therapy. ${ }^{19}$ In this problematic new meta-analysis, the authors looked at patients treated in clinical trials occurring 16-35 years ago for which $81 \%$ of patients did not receive taxanes, and no patients in these trials received anti-HER2 systemic therapy. Furthermore, the effect of potential omission of radiotherapy on LRR was unknown and not analyzed as data regarding this were incomplete and no information regarding the type of axillary surgery received and the potential impact on LRR was available. Thus, given these multiple factors, in addition to the critical need for state-ofthe-art imaging to select and surgically guide localization for breast-conserving surgery, and the lack of universal pathologic processing guidelines and reporting requirements in this meta-analysis, it remains unknown, yet unlikely, that the use of NST is associated with a potential for increased LRR in modern-day practice.

In the past, attempts were made to completely avoid surgery and just deliver chemotherapy and radiotherapy when patients had a complete clinical response to NST on physical examination. In the majority of studies, LRR rates were unacceptably high and occurred before our understanding of the importance of modern-day imaging, the use of image-guided biopsy to select patients, and the choice of optimal patients centered on the efficacy of improved agents based on molecular subtype. ${ }^{20}$ At this point, things are markedly different today and the therapeutic value of surgery becomes questionable once again when NST can eliminate both invasive and in situ carcinoma in up to $50 \%$ of patients, particularly when their subtypes are TNBC or HER2+ cancers. Breast imaging after NST lacks sufficient accuracy to determine which patients might be safe to move on to radiotherapy without the surgery. Recently, our group demonstrated that utilizing percutaneous biopsy after neoadjuvant therapy can accurately identify patients without residual disease. ${ }^{21}$ Combined biopsy demonstrated an accuracy of $98 \%$, false-negative rate of $5 \%$, and negative predictive value of $95 \%$ in predicting residual breast cancer. $^{21}$

The new paradigm of potential complete avoidance of surgery when there is complete eradication of disease with NST requires meticulous and precise image-guided biopsy of the tumor bed. ${ }^{16,21,22}$ Using the MD Anderson approach to optimize selection with extensive image-guided biopsy, approximately $48 \%$ of patients were found to have no evidence of disease in the breast after NST, and the rest of the cases demonstrated either residual carcinoma, atypia, the clip, and/or therapy changes consistent with representative sampling of the tumor bed. Furthermore, the pathologic response in the breast was concordant with the pathologic status in the axilla in approximately $98 \%$ of cases. $^{21,23}$ We know now that the false-negative cases result when there are $<12$ vacuum-assisted biopsy cores taken. Thus, to safely test the 'no surgery' concept in the ongoing MD Anderson study, a minimum of 12 cores of the original tumor bed and $<5 \mathrm{~cm}$ disease by initial breast imaging prior to NST are required. ${ }^{24}$ Regarding the management of the axilla when no breast surgery occurs, data from MD Anderson among 290 patients with TN/HER2+ breast cancer with T1-2 N0 disease and normal findings on nodal sonography at initial diagnosis showed that those with a pCR in the breast after NST demonstrated that none $(0 \%)$ had evidence of axillary lymph node metastases after NST. $^{23}$ Among 237 patients with fine-needle aspiration/core biopsy-documented N1 disease, $89.6 \%$ of patients with a breast pCR after NST had no evidence of axillary metastases, and $57.5 \%$ of patients without a breast pCR had 
residual axillary metastases. Thus, those with initial N0 disease will not have any nodal surgery. Patients with initial ultrasound biopsy-confirmed $\mathrm{N} 1$ disease are also eligible to participate in the study if biopsy does not demonstrate residual breast disease. However, these patients will require targeted axillary dissection to confirm no residual disease prior to radiotherapy. ${ }^{6}$

Finally, another approach that could be evaluated in clinical trials would be to de-escalate the use of radiotherapy, rather than the surgery. If the disease is completely eradicated with NST based on breast and nodal surgery, the additional benefit of breast and or nodal radiotherapy among cases with a pCR is unknown. This type of hypothetical clinical scenario is currently being tested in a randomized trial of no additional regional nodal and/or chest wall radiotherapy compared with standard radiotherapy among cases with documented nodal disease eradication after NST in the NSABP-51/RTOG 1304 trial. $^{25}$

There are currently multiple prospective, single-institution and cooperative groups testing the feasibility of accurately identifying patients who have no residual disease following NST with percutaneous image-guided biopsy, including the US cooperative group NRG-BR005 biopsy feasibility trial, which is open and accruing. ${ }^{26}$ When disease no longer exists in approximately $50 \%$ of selected patients receiving NST, we have a responsibility to identify these patients and test the potential of eliminating hypothetically unnecessary surgery. However, a critical takehome message regarding this new approach is that patients must do this only within well thought-out prospective clinical trials until the data show non-inferiority to our best available current treatments.

\section{REFERENCES}

1. Kuerer HM, Hunt KK, Newman LA, Ross MI, Ames FC, Singletary SE. Neoadjuvant chemotherapy in women with invasive breast carcinoma: conceptual basis and fundamental surgical issues. J Am Coll Surg. 2000;190(3):350-363.

2. Kuerer HM, Newman LA, Buzdar AU, et al. Pathologic tumor response in the breast following neoadjuvant chemotherapy predicts axillary lymph node status. Cancer J Sci Am. 1998;4(4):230-236.

3. Kuerer HM, Newman LA, Fornage BD, et al. Role of axillary lymph node dissection after tumor downstaging with induction chemotherapy for locally advanced breast cancer. Ann Surg Oncol. 1998;5(8):673-680.

4. Kuerer HM, Newman LA, Smith TL, et al. Clinical course of breast cancer patients with complete pathologic primary tumor and axillary lymph node response to doxorubicin-based neoadjuvant chemotherapy. J Clin Oncol. 1999;17(2):460-469.

5. Kuerer HM, Sahin AA, Hunt KK, et al. Incidence and impact of documented eradication of breast cancer axillary lymph node metastases before surgery in patients treated with neoadjuvant chemotherapy. Ann Surg. 1999;230(1):72-78.
6. Caudle AS, Yang WT, Krishnamurthy S, et al. Improved axillary evaluation following neoadjuvant therapy for patients with nodepositive breast cancer using selective evaluation of clipped nodes: implementation of targeted axillary dissection. J Clin Oncol. 2016;34(10):1072-1078.

7. Fisher B, Bryant J, Wolmark N, et al. Effect of preoperative chemotherapy on the outcome of women with operable breast cancer. J Clin Oncol. 1998;16(8):2672-2685.

8. Shin K, Caudle AS, Kuerer HM, et al. Radiologic Mapping for targeted axillary dissection: needle biopsy to excision. AJR Am J Roentgenol. 2016;207(6):1372-1379.

9. Hennessy BT, Hortobagyi GN, Rouzier R, et al. Outcome after pathologic complete eradication of cytologically proven breast cancer axillary node metastases following primary chemotherapy. J Clin Oncol. 2005;23(36):9304-9311.

10. Houssami N, Macaskill P, von Minckwitz G, Marinovich ML, Mamounas E. Meta-analysis of the association of breast cancer subtype and pathologic complete response to neoadjuvant chemotherapy. Eur J Cancer. 2012;48(18):3342-3354.

11. von Minckwitz G, Untch M, Blohmer JU, et al. Definition and impact of pathologic complete response on prognosis after neoadjuvant chemotherapy in various intrinsic breast cancer subtypes. J Clin Oncol. 2012;30(15):1796-1804.

12. Gianni L, Pienkowski T, Im YH, et al. Efficacy and safety of neoadjuvant pertuzumab and trastuzumab in women with locally advanced, inflammatory, or early HER2-positive breast cancer (NeoSphere): a randomised multicentre, open-label, phase 2 trial. Lancet Oncol. 2012;13(1):25-32.

13. Dominici LS, Negron Gonzalez VM, Buzdar AU, et al. Cytologically proven axillary lymph node metastases are eradicated in patients receiving preoperative chemotherapy with concurrent trastuzumab for HER2-positive breast cancer. Cancer. 2010;116(12):2884-2889.

14. Masuda N, Lee SJ, Ohtani S, et al. Adjuvant capecitabine for breast cancer after preoperative chemotherapy. $N$ Engl J Med. 2017;376(22):2147-2159.

15. Murphy BL, Day CN, Hoskin TL, Haberman EB, Boughey JC. Neoadjuvant chemotherapy use in breast cancer is greatest in excellent responders: Triple-negative and HER2+ subtypes. Ann Surg Oncol; 2018. https://doi.org/10.1245/s10434-018-6531-5.

16. Rauch GM, Kuerer HM, Adrada B, et al. Biopsy feasibility trial for breast cancer pathologic complete response detection after neoadjuvant chemotherapy: imaging assessment and correlation endpoints. Ann Surg Oncol. 2018;25(7):1953-1960.

17. Mauri D, Pavlidis N, Ioannidis JP. Neoadjuvant versus adjuvant systemic treatment in breast cancer: a meta-analysis. J Natl Cancer Inst. 2005;97(3):188-194.

18. Mieog JS, van der Hage JA, van de Velde CJ. Preoperative chemotherapy for women with operable breast cancer. Cochrane Database Syst Rev. 2007;2:CD005002.

19. Early Breast Cancer Trialists' Collaborative G. Long-term outcomes for neoadjuvant versus adjuvant chemotherapy in early breast cancer: meta-analysis of individual patient data from ten randomised trials. Lancet Oncol. 2018;19(1):27-39.

20. van la Parra RF, Kuerer HM. Selective elimination of breast cancer surgery in exceptional responders: historical perspective and current trials. Breast Cancer Res. 2016;18(1):28.

21. Kuerer HM, Rauch GM, Krishnamurthy S, et al. A clinical feasibility trial for identification of exceptional responders in whom breast cancer surgery can be eliminated following neoadjuvant systemic therapy. Ann Surg. 2018;267(5):946-951.

22. van la Parra RFD, Tadros AB, Checka CM, et al. Baseline factors predicting a response to neoadjuvant chemotherapy with implications for non-surgical management of triple-negative breast cancer. Br J Surg. 2018;105(5):535-543. 
23. Tadros AB, Yang WT, Krishnamurthy S, et al. Identification of patients with documented pathologic complete response in the breast after neoadjuvant chemotherapy for omission of axillary surgery. JAMA Surg. 2017;152(7):665-670.

24. Kuerer H. Eliminating breast cancer surgery in exceptional responders with neoadjuvant systemic therapy [ClinicalTrials. gov]; 2018. https://clinicaltrials.gov/ct2/show/NCT02945579. Accessed 5 July 2018.
25. Caudle AS, Kuerer HM. Targeting and limiting surgery for patients with node-positive breast cancer. BMC Med. 2015; 13:149.

26. Kuerer HM, Vrancken Peeters M, Rea DW, Basik M, De Los Santos J, Heil J. Nonoperative management for invasive breast cancer after neoadjuvant systemic therapy: conceptual basis and fundamental international feasibility clinical trials. Ann Surg Oncol. 2017;24(10):2855-2862. 